\title{
Increased p16 and p53 protein expression predicts poor prognosis in mucosal melanoma
}

\author{
Hanbin Chen ${ }^{1, *}$, Yangyang Li ${ }^{2,}$, Yin Long ${ }^{3}$, Erjiang Tang ${ }^{3}$, Rongrong Wang ${ }^{2}$, Kate \\ Huang $^{2, *}$, Congying Xie ${ }^{1, *}$ and Guorong Chen ${ }^{2, *}$ \\ ${ }^{1}$ Department of Radiotherapy and Chemotherapy, The First Affiliated Hospital of Wenzhou Medical University, Wenzhou, \\ Zhejiang, China \\ ${ }^{2}$ Department of Pathology, The First Affiliated Hospital of Wenzhou Medical University, Wenzhou, Zhejiang, China \\ ${ }^{3}$ Center for Translational Medicine, Yangpu Hospital, Tongji University School of Medicine, Yangpu, Shanghai, China \\ *These authors have contributed equally to this work \\ Correspondence to: Kate Huang, email: handyman2002@163.com \\ Congying Xie, email: wzxiecongying@163.com \\ Guorong Chen, email: chengr1978@aliyun.com \\ Keywords: 16 protein, p53 protein, mucosal melanoma, prognosis of mucosal melanoma \\ Received: April 07, $2017 \quad$ Accepted: May 10, $2017 \quad$ Published: June 05, 2017 \\ Copyright: Chen et al. This is an open-access article distributed under the terms of the Creative Commons Attribution License 3.0 \\ (CC BY 3.0), which permits unrestricted use, distribution, and reproduction in any medium, provided the original author and source \\ are credited.
}

\section{ABSTRACT}

Primary mucosal melanoma (MM) is a rare, and aggressive, neoplasm with a poor prognosis. To date, few prognostic markers of MM have been well-defined. The aim of this study is to clarify the prognostic value of p53 and p16 proteins in predicting the clinical outcome of Chinese patients with MM.

A total of $59 \mathrm{MM}$ samples were contained from biopsy specimens, and, expressions of p53 and p16 proteins were assessed by immunohistochemistry. Cox regression analysis was performed to investigate the association of these proteins with the overall survival of MM patients. Increased p16 expression was significantly associated with reduced survival at three years $(P=0.039)$. Increased p53 expression correlates with reduced one-year $(P=0.025)$, and, two-year survival $(P=0.037)$.

Increased p53 and p16 protein expression may be helpful prognostic indicators for the management of these patients.

\section{INTRODUCTION}

Mucosal Melanoma (MM) is a rare subtype of malignant melanoma representing approximately $1.3 \%$ of all melanomas [1-4]. Primary MM may arise from the head and neck $(55 \%)$, female genital tract (18\%), anorectal (24\%), or urinary tract (3\%), respectively [5]. Although the prevalence of this disease is relatively low, it has become increasingly clear that primary MM leads to worse outcome than other subtypes of melanoma. According to the data from a large, US registry study, the mean five-year overall survival rate for primary MM is only $25 \%$; substantially lower than the $80 \%$ survival rate for cutaneous melanoma and $75 \%$ for ocular melanoma
[5]. Stratified by anatomic sites, five-year overall survival rate for primary $\mathrm{MM}$ varies with $30 \%$ for the head and neck, $10 \%$ for the female lower genital tract and $20 \%$ for the anorectum [5]. This suggests that primary MM is a biologically-heterogeneous disease with wide variations in prognosis.

p53 is a tumor suppressor gene that plays a key role in maintaining genome stability and regulating cell cycle, apoptosis, differentiation and senescence [6]. It operates as a redox-sensitive transcription factor where ROS modifies p53 cysteine residues that affects transcriptional activity, and, stimulates a number of signaling pathways (APE-1/Ref1, ASK1/-p38, MAPK, PTPs-PKC8) [7]. p53 generally mutates in cancer leading to dysregulation 
of downstream genes $[8,9]$. Many tumor-derived p53 mutants cause inactivation of $\mathrm{p} 53$ inducible protein 3 leading to accumulation of $\mathrm{p} 53$ and poor prognosis in a wide range of cancers $[10,11]$. High levels of p53 also induce downregulation of superoxide dismutase that promotes apoptosis [12].

p16 is a kinase inhibitor encoded by the INK4a/ CDKN2A gene. It binds to cyclin-dependent kinases (CDKs) CDK4 and CDK6, inhibits the phosphorylation of RB, resulting in cell cycle arrest, and, suppressing cell proliferation [13]. p16 overexpression occurs at the invasive margin in endometrial, colorectal and basal cell carcinoma suggesting a role in invasion [14-17]. It also plays a role in apoptosis and angiogenesis; restoring p16 Ink-4a results in down-regulation of vascular endothelial growth factor in different cell lines, and, inhibition of angiogenesis in malignant gliomas [18]. Interestingly, overexpression of p16 has been observed in a number of cancer cell lines in a p53 dependent $[19,20]$ or p53independent manner $[21,22]$. Taken together blocking cell proliferation, invasion and angiogenesis suggests a role for p16 ${ }^{\text {Ink-4a }}$ as a "universal suppressor" in cancer cells.

Similarly, p16 is also significantly associated with cancer prognosis $[23,24]$. Most malignant tumors over-expressing p16 Ink-4a do not demonstrate constant overexpression suggesting that they may bypass cell senescence in malignant disease. p16 overexpression in colon adenocarcinoma correlates with features of poor prognosis, and, in breast cancer, $20 \%$ of tumors overexpress p16 correlating with high grade and negative estrogen receptor status [22]. In that study p16 overexpression was also associated with p53 expression, vascular invasion and lack of progesterone receptors.

TNM staging is proposed by NCCN guidelines to predict prognosis and guide therapy for MM. Although this staging system takes into account the impact of tumor size and its metastasis to lymph node and other organs on prognosis, it is insufficient to guide individualized treatment due to a lack of prognostic markers. Therefore, it is important to identify factors capable of stratifying MM patients and predicting their outcomes. The purpose of this study is to assess the predictive value of p16 and p53 in Chinese MM patients.

\section{RESULTS}

\section{Characteristics of MM patients}

The clinical data of the $59 \mathrm{MM}$ patients are summarized in Table 1 . Overall, the median age of the patients is 73 years (range 31 to 96). There were 26 female and 33 male patients recruited to the study. The primary lesions were distributed in the head and neck $(47 / 59,79.7 \%)$, gastrointestinal system $(8 / 59,13.6 \%)$, genitourinary systems $(3 / 59,5.1 \%)$ lung $(1 / 59,1.7 \%)$, respectively. Head and neck MM patients consisted of
37 cases in the nasal cavity, 5 cases in the paranasal sinuses, 3 cases in the ocular orbit, and, 2 cases in the oral cavity.

\section{Protein expression of p16 and p53 in MM tissues}

Expression of p16 was detected in both the nucleus and cytoplasm, whereas $\mathrm{p} 53$ protein was confined to the nucleus (Figure 1). Patients with low p16 expression accounted for $47.5 \%$, compared with $52.5 \%$ patients with high expression of p16 (Table 1). High expression of p53 was scored in $20.3 \%(12 / 59)$ of MM patients, which is much lower than that of patients with low expression of p53. Neither p16 nor p53 significantly correlated with the clinical characteristics.

\section{Association of p53 and p16 with survival}

Patients with high p53 expression had unfavorable one-year (HR, 3.26, 95\% CI, 1.16-9.17, $\mathrm{p}=0.025$ ), twoyear $(\mathrm{HR}, 2.41,95 \% \mathrm{CI}, 1.05-5.54, \mathrm{p}=0.037)$ and threeyear $(\mathrm{HR}, 2.09,95 \% \mathrm{CI}, 0.97-4.51, \mathrm{p}=0.061)$ survival rates (Figure 2). Patients at high risk showed shorter mean survival times (12 months) in contrast to patients at low risk with longer mean survival times (33 months).Patients with a low expression of p16, had better three-year survival compared to patients with high p16 expression (HR, 2.12, 95\%CI, 1.04-4.32, $\mathrm{P}=0.039$ ) (Figure 2). The mean survival time of patients with low p16 expression was over 36 months compared to 22 months for patients with high p16 expression. Accordingly, patients with high p16 showed a trend toward poor one-year survival rates (HR, 2.78, 95\% CI, 0.88-8.73, $\mathrm{p}=0.081$ ) and two-year (HR, $1.69,95 \% \mathrm{CI}, 0.79-3.66, \mathrm{p}=0.181$ ).

\section{DISCUSSION}

Increased expression of p53 and p16 were significantly associated with reduced overall survival in a cohort of 59 Chinese patients diagnosed with mucosal melanoma. For p16 this was limited to reduced overall, three year survival, and, for p53 it was reduced to one and two year survival (with a trend towards worse three year survival that did not reach statistical significance). These results are comparable with other reports of p16 and p53 overexpression in the literature though there are few reports discussing this particular tumor.

p53 expression appears abnormal in cutaneous and uveal melanomas [25-27]. Similar to cutaneous melanoma, high p53 expression was found in $20.3 \%$ (12/59) of MM samples in our study [28]. In general, over-expression of $\mathrm{p} 53$ in cancer is attributed to a series of p53 mutations which either result in dominantnegative effects over wild-type p53, or, acquire new activities contributing to tumor progression and drug resistance $[29,10,30,31]$. Previously, Cox regression 
Table 1: Characteristics of patients

\begin{tabular}{|c|c|c|c|c|c|c|c|c|}
\hline \multirow[t]{2}{*}{ Characteristics } & & \multirow[t]{2}{*}{ Number of patients(\%) } & \multicolumn{3}{|c|}{ p53 } & \multicolumn{3}{|c|}{ p16 } \\
\hline & & & Low $^{\#}$ & High $^{\# \#}$ & Pvalue* & Low $^{\#}$ & High $^{\# \#}$ & P value* \\
\hline Total patients & & 59 & $47(79.7)$ & $12(20.3)$ & & $28(47.5)$ & $31(52.5)$ & \\
\hline \multirow{3}{*}{$\begin{array}{l}\text { Age (year), } \\
\text { median(range) }\end{array}$} & & $73(31-96)$ & & & & & & \\
\hline & $\leq 73$ & $30(50.8)$ & $23(76.7)$ & $7(23.3)$ & & $15(50.0)$ & $15(50.0)$ & \\
\hline & $>73$ & $29(49.2)$ & $24(82.8)$ & $5(17.2)$ & 0.748 & $13(44.8)$ & $16(55.2)$ & 0.796 \\
\hline \multicolumn{9}{|l|}{ Gender, N (\%) } \\
\hline & Male & $33(55.9)$ & $28(84.8)$ & $5(15.2)$ & & $16(48.5)$ & $17(51.5)$ & \\
\hline & Female & $26(44.1)$ & $19(73.1)$ & $7(26.9)$ & 0.336 & $12(46.2)$ & $14(53.8)$ & 1 \\
\hline \multicolumn{9}{|l|}{ Anatomic sites } \\
\hline & Head and neck & 47(79.7) & $38(80.9)$ & $9(19.1)$ & & $21(44.7)$ & $26(55.3)$ & \\
\hline & Gastrointestine & $8(13.5)$ & $5(62.5)$ & $3(37.5)$ & & $5(62.5)$ & $3(37.5)$ & \\
\hline & Genitourinary & $3(5.1)$ & $3(100.0)$ & 0 & & $2(66.7)$ & $1(33.3)$ & \\
\hline & Lung & $1(1.7)$ & $1(100.0)$ & 0 & 0.486 & 0 & $1(100.0)$ & 0.542 \\
\hline \multicolumn{9}{|l|}{ Survival, N(\%) } \\
\hline & Alive & $19(32.2)$ & $17(89.5)$ & $2(10.5)$ & & $10(52.6)$ & $9(47.4)$ & \\
\hline & Death & $40(67.8)$ & $30(75.0)$ & $10(25.0)$ & 0.303 & $18(45.0)$ & $22(55.0)$ & 0.781 \\
\hline \multicolumn{9}{|l|}{ One-year survival } \\
\hline & Survival & $40(70.2)$ & $35(87.5)$ & $5(12.5)$ & $0.025^{*}$ & $23(57.5)$ & $17(42.5)$ & 0.081 \\
\hline \multicolumn{9}{|l|}{ Two-year survival } \\
\hline & Survival & $23(46.0)$ & $20(87.0)$ & $3(13.0)$ & $0.037 *$ & $13(56.5)$ & $10(43.5)$ & 0.181 \\
\hline \multicolumn{9}{|c|}{ Three-year survival } \\
\hline & Survival & $13(28.3)$ & $11(84.6)$ & $2(15.4)$ & 0.061 & $9(69.2)$ & $4(30.6)$ & $0.039 *$ \\
\hline
\end{tabular}

analysis showed that cytoplasmic p16 expression significantly correlated with poor survival in high-grade astrocytomas [32]. Similarly, another large retrospective study of 2197 breast cancer patients showed that strong p16 expression was significantly linked to shortened survival time [33]. Accordingly, high p16 expression was demonstrated to be significantly associated with a low three-year survival rate and showed a trend toward poor one-year and two-year survival rates (Figure 2).

It is important to bear in mind that this study used biopsy specimens to determine the expression of these proteins. There was not a study of specimens from a fullstaging procedure so we cannot comment on the size, nature and extent of the initial tumor, in relation to the clinical outcomes. Also these patients only received adjuvant radiotherapy or chemotherapy if they developed metastatic disease so we cannot comment on the relationship between p16 and p53 and their importance in radiosensitive tumors.
A previous study in Korea reported an overall mean survival time of MM of 24.4 months, with two-year and five-year survival rates at $50.7 \%$ and $25.5 \%$ respectively [34]. We observed that positive p16 staining accumulated in both the nucleus and cytoplasm (Figure 1), consistent with other studies in $\mathrm{MM}$ and breast cancer [35, 36]. Intriguingly, p16 was detected in over $50 \%$ of samples in our study; relatively higher than the $25 \%$ detection rate in Western patients [37]. This suggests that the regulatory, molecular mechanisms leading to aberrant p16 expression may differ between races. Clearly we need to complete the follow-up of this group of patients to achieve a final conclusion about the utility of these markers in this rare tumor. There will be other groups of patients with different cancers that can be fully staged, where this combination of markers may provide useful predictive information about prognosis. 

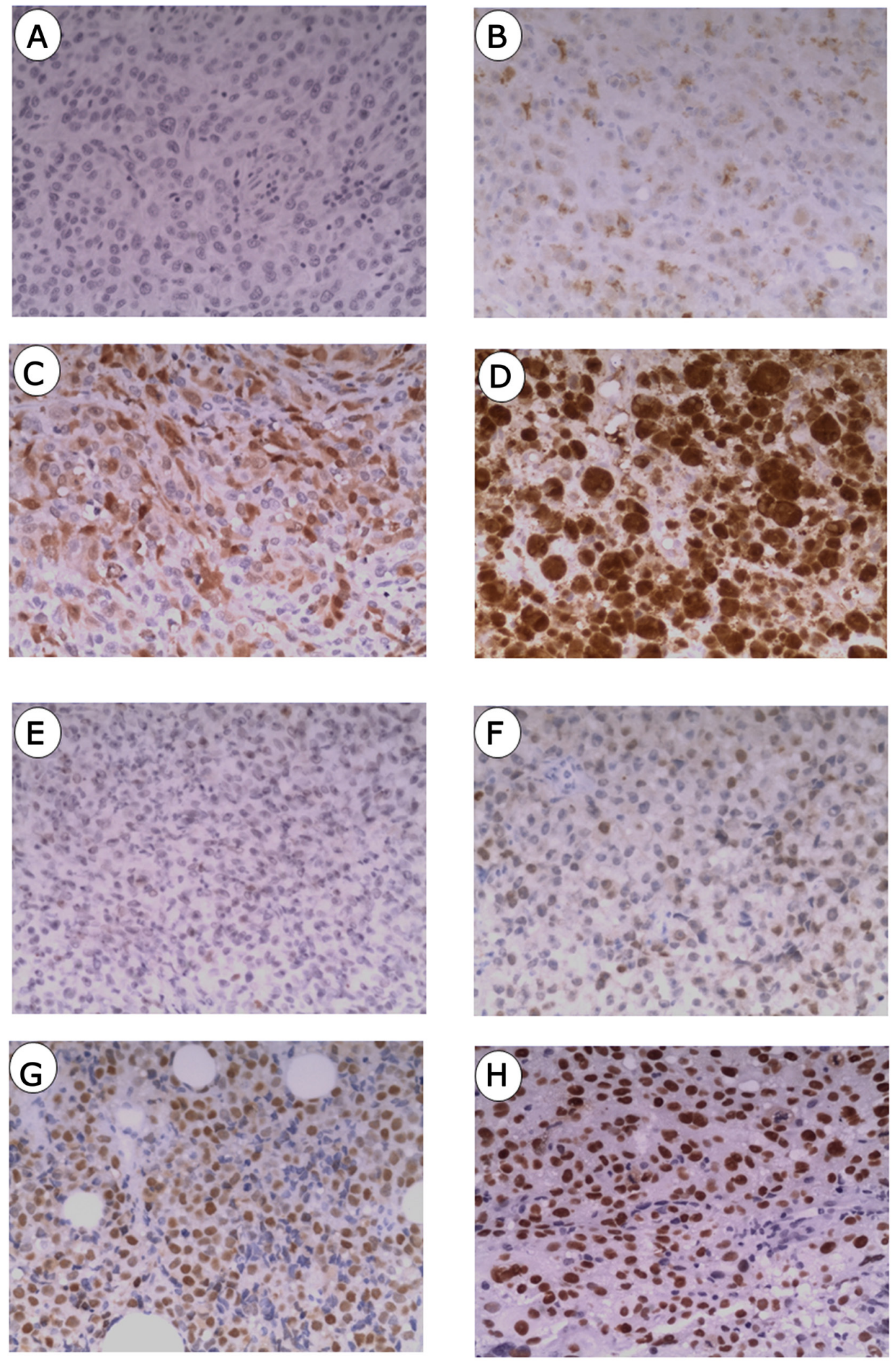

Figure 1: Immunostainings of p16 and p53 in mucosal melanoma. (A) Negative staining of p16. (B) Weak staining of p16. (C) Moderate staining of p16. (D) Strong staining of p16. (E) Negative staining of p53. (F) Weak staining of p53. (G) Moderate staining of p53 protein. (H) Strong staining of p53. 

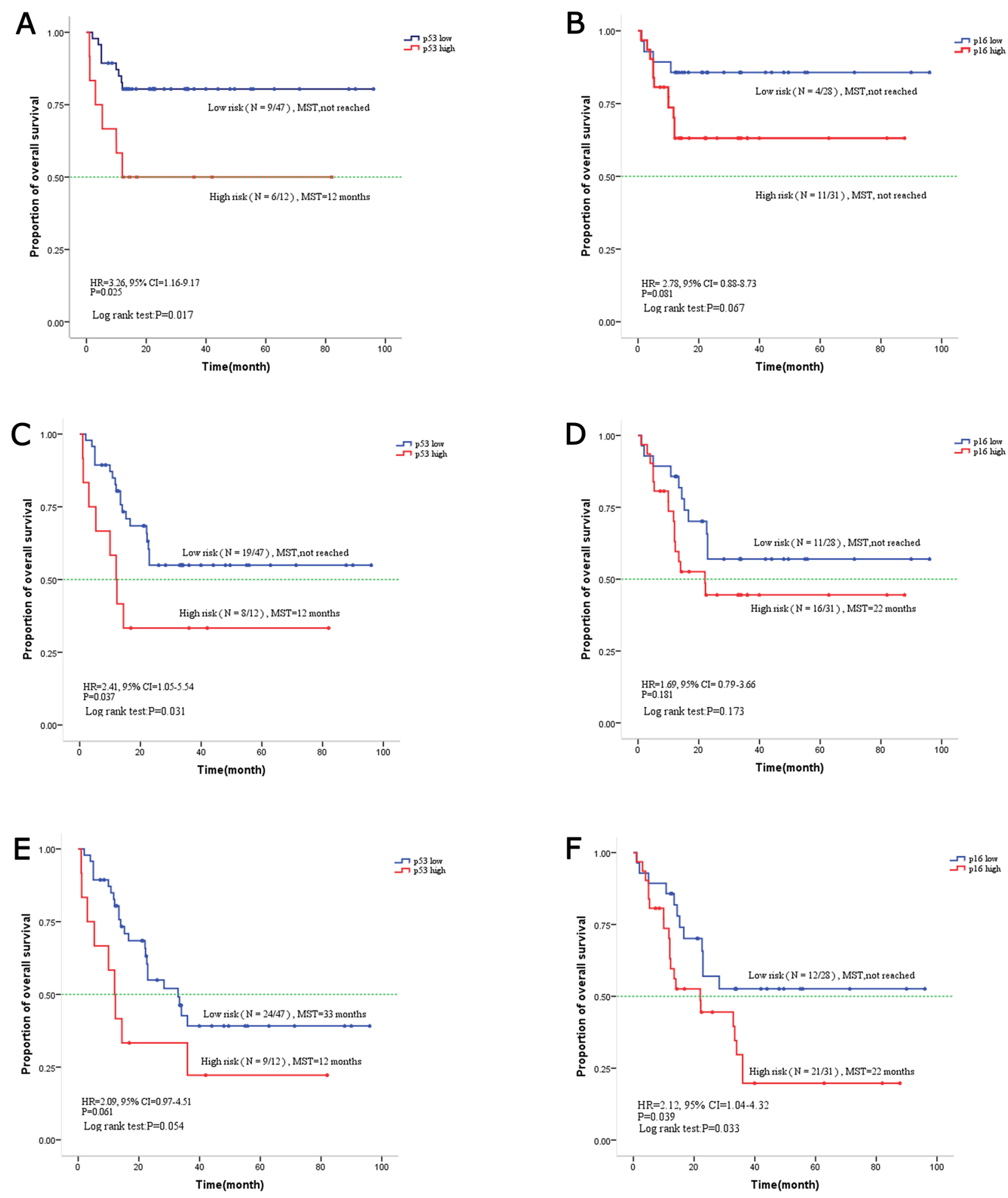

Figure 2: Kaplan-Meier curve for overall survival based on p16 and p53 expression in mucosal melanoma. (A) One-year overall survival based on p53. (C) Two-year overall survival based on p53. (E) Three-year overall survival based on p53. (B) One-year overall survival based on p16. (D) Two-year overall survival based on p16. (F) Three-year overall survival based on p16. 


\section{PATIENTS AND METHODS}

\section{Patients and tumor tissue samples}

The current study included a total of 59 patients who were diagnosed as $\mathrm{MM}$ and underwent surgery between 2000 and 2015 in the First Affiliated Hospital of Wenzhou Medical University in Eastern China. Patient eligibility included a histopathologic diagnosis of MM, and, a complete set of clinical and follow-up information. Overall survival time was assessed beginning from the time of initial diagnosis to the last follow-up or, date of death. Pathologic observations were evaluated by two pathologists independently using the existing hematoxylin-eosin(H\&E) slides from routine diagnostics. The study was reviewed and approved by the institutional review board.

\section{Histology and Immunohistochemistry}

All surgical specimens from MM patients were studied by gross and microscopic examination. Gross appearances of MM samples included large polypoidal masses, with or without, melanin pigment. All tissue samples sent were fixed in $4 \%$ formaldehyde. Specimens were subject to subsequent steps of dehydration, paraffin embedding, sectioning, hematoxylin, eosin (HE) staining, and light microscopy. Histologic examination revealed intratumoral heterogeneity including epithelioid, spindled, and small cell cytomorphology. Melanoma was diagnosed directly when the tumor cells were melanin-rich, and, had immunohistochemical staining for HMB45, Melan A, S100, CK and Ki67 as previous described [38, 39]. Immunohistochemical staining was performed using the p53 primary antibody (Maxim, China) and p16 primary antibody (ZSGB-BIO, China) in the 59 samples according to the manufacturer's instruction.

\section{Immunohistochemical scoring and analysis}

The staining intensity was scored using the following scale: no staining (0), weak (1), moderate (2), and strong (3). The staining intensity scores were then evaluated by two pathologists. Immunoreactive score (IRS) was then used to determine the staining level by staining intensity, and this IRS was used to grade the protein expression. IRS over 1 was considered as high expression while IRS below or equal to 1 was considered as low expression.

\section{Statistical analysis}

The statistical analysis was done using $\mathrm{R}$ program. The Kaplan-Meier method was utilized to graph the survival curves. The log-rank test was used to analyze the significant difference of Kaplan-Meier curves. The overall risk of death was estimated as hazard ratios (HRs) and a 95\% confidence interval (CI) using the Cox Regression Model. The $\chi 2$ test was utilized to determine the difference of clinicopathologic features between pairs of groups. In all analyses, P-value $\leq 0.05$ was considered statistically significant.

\section{Author contributions}

Study design: H C, Y-Y L. Clinical studies: Y L,C X. Experimental studies: K H, R W. Data analysis: E T. Manuscript writing: Y L, G C.

\section{ACKNOWLEDGMENTS}

The patients' data and the pathological section were evaluated and provided by the Department of Pathology, the First Affiliated Hospital of Wenzhou Medical University, Wenzhou, Zhejiang, China.

\section{CONFLICTS OF INTEREST}

We state no conflicts of interest. The funding bodies had no role in study design, data collection and analysis, decision to publish or preparation of the manuscript. The contents have not been published by or submitted to any other journals. No part of this manuscript has been included in any other manuscript.

\section{FUNDING}

This work was supported by the Zhejiang Science \& Technology (2013C24031) and the Wenzhou Scientific and Technological Project (Y20130001).

\section{REFERENCES}

1. Curtin JA, Fridlyand J, Kageshita T, Patel HN, Busam KJ, Kutzner H, Cho KH, Aiba S, Brocker EB, LeBoit PE, Pinkel D, Bastian BC. Distinct sets of genetic alterations in melanoma. N Engl J Med 2005; 353:2135-2147.

2. Guo J, Qin S, Liang J, Lin T, Si L, Chen X, Chi Z, Cui C, Du N, Fan Y, Gu K, Li F, Li J, et al. Chinese guidelines on the diagnosis and treatment of melanoma (2015 edition). Chin Clin Oncol. 2016; 5:57.

3. Kong Y, Si L, Zhu Y, Xu X, Corless CL, Flaherty KT, Li L, Li H, Sheng X, Cui C, Chi Z, Li S, Han M, et al. Largescale analysis of KIT aberrations in Chinese patients with melanoma. Clin Cancer Res. 2011; 17:1684-1691.

4. Mikkelsen LH, Larsen AC, von Buchwald C, Drzewiecki KT, Prause JU, Heegaard S. Mucosal malignant melanoma - a clinical, oncological, pathological and genetic survey. APMIS. 2016; 124:475-486.

5. Chang AE, Karnell LH, Menck HR. The National Cancer Data Base report on cutaneous and noncutaneous melanoma: a summary of 84,836 cases from the past decade. The American College of Surgeons Commission 
on Cancer and the American Cancer Society. Cancer. 1998; 83:1664-1678.

6. Vousden $\mathrm{KH}$, Prives C. Blinded by the light: the growing complexity of p53. Cell. 2009; 137:413-431.

7. Vurusaner B, Poli G, Basaga H. Tumor suppressor genes and ROS: complex networks of interactions. Free Radic Biol Med. 2012; 52:7-18.

8. Ladelfa MF, Toledo MF, Laiseca JE, Monte M. Interaction of p53 with tumor suppressive and oncogenic signaling pathways to control cellular reactive oxygen species production. Antioxid Redox Signal. 2011; 15:1749.

9. Kotsinas A, Aggarwal V, Tan EJ, Levy B, Gorgoulis VG. PIG3: a novel link between oxidative stress and DNA damage response in cancer. Cancer Lett. 2011; 327:97-102.

10. Kim HS, Yom CK, Kim HJ, Lee JW, Sohn JH, Kim JH, Park YL, Ahn SH. Overexpression of p53 is correlated with poor outcome in premenopausal women with breast cancer treated with tamoxifen after chemotherapy. Breast Cancer Res Treat. 2010; 121:777-788.

11. Dong Y, Walsh MD, McGuckin MA, Cummings MC, Gabrielli BG, Wright GR, Hurst T, Khoo SK, Parsons PG. Reduced expression of retinoblastoma gene product (pRB) and high expression of p53 are associated with poor prognosis in ovarian cancer. Int J Cancer. 1997; 74:407-415.

12. Pani G, Galeotti T. Role of MnSOD and p66shc in mitochondrial response to p53. Antioxid Redox Signal. 2011; 15:1715.

13. LaPak KM, Burd CE. The molecular balancing act of p16(INK4a) in cancer and aging. Mol Cancer Res. 2014; 12:167-183.

14. Jung A, Schrauder M, Oswald U, Knoll C, Sellberg P, Palmqvist R, Niedobitek G, Brabletz T, Kirchner T. The invasion front of human colorectal adenocarcinomas shows co-localization of nuclear $\beta$-catenin, cyclin D1, and p16INK4A and is a region of low proliferation. Am J Pathol. 2001; 159:1613-1617.

15. Natarajan E, Saeb M, Crum CP, Woo SB, Mckee PH, Rheinwald JG. Co-expression of p16 INK4A and laminin $5 \gamma 2$ by microinvasive and superficial squamous cell carcinomas in vivo and by migrating wound and senescent keratinocytes in culture. Am J Pathol. 2003; 163:477.

16. Svensson S, Nilsson K, Ringberg A, Landberg G. Invade or proliferate? Two contrasting events in malignant behavior governed by p16(INK4a) and an intact Rb pathway illustrated by a model system of basal cell carcinoma. Cancer Res. 2003; 63:1737-1742.

17. Horrée $\mathrm{N}$, van Diest PJ, Sie-Go DM, Heintz AP. The invasive front in endometrial carcinoma: higher proliferation and associated derailment of cell cycle regulators. Human Pathol. 2007; 38:1232-1238.

18. Harada H, Nakagawa K, Iwata S, Saito M, Kumon Y, Sakaki S, Sato K, Hamada K. Restoration of wild-type p16 down-regulates vascular endothelial growth factor expression and inhibits angiogenesis in human gliomas. Cancer Res. 1999; 59:3783-3789.

19. Spitz F. Down-regulation of bcl-2 is associated with p16INK4-mediated apoptosis in non-small cell lung cancer cells. Oncogene. 2000; 19:1589.

20. Katsuda K, Kataoka M, Uno F, Murakami T, Kondo T, Roth JA, Tanaka N, Fujiwara T. Activation of caspase-3 and cleavage of $\mathrm{Rb}$ are associated with p16-mediated apoptosis in human non-small cell lung cancer cells. Oncogene. 2002; 21:2108-2113.

21. Roig JM. Adenovirus-mediated wt-p16 reintroduction induces cell cycle arrest or apoptosis in pancreatic cancer. Cancer Gene Ther. 2001; 8:740-750.

22. Modesitt SC, Ramirez P, Zu Z, Bodurkabevers D, Gershenson D, Wolf JK. In vitro and in vivo adenovirusmediated p53 and p16 tumor suppressor therapy in ovarian cancer. Clin Cancer Res. 2001; 7:1765-1772.

23. Salazar CR, Anayannis N, Smith RV, Wang Y, Haigentz M Jr, Garg M, Schiff BA, Kawachi N, Elman J, Belbin TJ, Prystowsky MB, Burk RD, Schlecht NF. Combined P16 and human papillomavirus testing predicts head and neck cancer survival. Int J Cancer. 2014; 135:2404-2412.

24. Zhao N, Ang MK, Yin XY, Patel MR, Fritchie K, Thorne L, Muldrew KL, Hayward MC, Sun W, Wilkerson MD, Chera BS, Hackman T, Zanation AM, et al. Different cellular p16(INK4a) localisation may signal different survival outcomes in head and neck cancer. Br J Cancer. 2012; 107:482-490.

25. Coupland SE, Anastassiou G, Stang A, Schilling H, Anagnostopoulos I, Bornfeld N, Stein H. The prognostic value of cyclin D1, p53, and MDM2 protein expression in uveal melanoma. J Pathol. 2000; 191:120-126.

26. Gelsleichter L, Gown AM, Zarbo RJ, Wang E, Coltrera MD. p53 and mdm-2 expression in malignant melanoma: an immunocytochemical study of expression of p53, mdm2 , and markers of cell proliferation in primary versus metastatic tumors. Mod Pathol. 1995; 8:530-535.

27. Gwosdz C, Scheckenbach K, Lieven O, Reifenberger J, Knopf A, Bier H, Balz V. Comprehensive analysis of the p53 status in mucosal and cutaneous melanomas. Int $\mathrm{J}$ Cancer. 2006; 118:577-582.

28. Straume O, Akslen LA. Alterations and prognostic significance of $\mathrm{p} 16$ and $\mathrm{p} 53$ protein expression in subgroups of cutaneous melanoma. Int J Cancer. 1997; 74:535-539.

29. Parrales A, Iwakuma T. Targeting oncogenic mutant $\mathrm{p} 53$ for cancer therapy. Front Oncol. 2015; 5:288.

30. Bali A, O'Brien PM, Edwards LS, Sutherland RL, Hacker NF, Henshall SM. Cyclin D1, p53, and p21Waf1/Cip1 expression is predictive of poor clinical outcome in serous epithelial ovarian cancer. Clin Cancer Res. 2004; 10:5168-5177. 
31. Cleven AH, Nardi V, Ok CY, Goswami M, Dal Cin P, Zheng Z, Iafrate AJ, Abdul Hamid MA, Wang SA, Hasserjian RP. High p53 protein expression in therapy-related myeloid neoplasms is associated with adverse karyotype and poor outcome. Mod Pathol. 2015; 28:552-563.

32. Arifin MT, Hama S, Kajiwara Y, Sugiyama K, Saito T, Matsuura S, Yamasaki F, Arita K, Kurisu K. Cytoplasmic, but not nuclear, p16 expression may signal poor prognosis in high-grade astrocytomas. J Neurooncol. 2006; 77:273-277.

33. Lebok P, Roming M, Kluth M, Koop C, Ozden C, Taskin B, Hussein K, Lebeau A, Witzel I, Wolber L, Geist S, Paluchowski P, Wilke C, et al. p16 overexpression and 9p21 deletion are linked to unfavorable tumor phenotype in breast cancer. Oncotarget. 2016; 7:81322-81331. doi: 10.18632/oncotarget.13227.

34. Kim HS, Kim EK, Jun HJ, Oh SY, Park KW, Lim DH, Lee SI, Kim JH, Kim KM, Lee DH, Lee J. Noncutaneous malignant melanoma: a prognostic model from a retrospective multicenter study. BMC Cancer. 2010; 10:167.

35. Gray-Schopfer VC, Cheong SC, Chong H, Chow J, Moss T, Abdel-Malek ZA, Marais R, Wynford-Thomas D, Bennett
DC. Cellular senescence in naevi and immortalisation in melanoma: a role for p16? Br J Cancer. 2006; 95:496-505.

36. Emig R, Magener A, Ehemann V, Meyer A, Stilgenbauer F, Volkmann M, Wallwiener D, Sinn HP. Aberrant cytoplasmic expression of the 16 protein in breast cancer is associated with accelerated tumour proliferation. Br J Cancer. 1998; 78:1661-1668.

37. Prasad ML, Patel SG, Shah JP, Hoshaw-Woodard S, Busam KJ. Prognostic significance of regulators of cell cycle and apoptosis, p16(INK4a), p53, and bcl-2 in primary mucosal melanomas of the head and neck. Head Neck Pathol. 2012; 6:184-190.

38. Zheng J, Mo H, Ma S, Wang Z. Clinicopathological findings of primary esophageal malignant melanoma: report of six cases and review of literature. Int J Clin Exp Pathol. 2014; 7:7230-7235.

39. Thompson LD, Wieneke JA, Miettinen M. Sinonasal tract and nasopharyngeal melanomas: a clinicopathologic study of 115 cases with a proposed staging system. Am J Surg Pathol. 2003; 27:594-611. 